\title{
Image guidance based on MRI for spinal interstitial laser thermotherapy: technical aspects and accuracy
}

\author{
Claudio E. Tatsui, MD, ${ }^{1}$ Clarissa N. G. Nascimento, MD, ${ }^{2}$ Dima Suki, PhD, ${ }^{1}$ Behrang Amini, MD, PhD, ${ }^{3}$ \\ Jing Li, MD, ${ }^{4}$ Amol J. Ghia, MD, ${ }^{4}$ Jonathan G. Thomas, MD, ${ }^{1}$ R. Jason Stafford, PhD, ${ }^{5}$ \\ Laurence D. Rhines, MD, ${ }^{1}$ Juan P. Cata, MD, ${ }^{6}$ Ashok J. Kumar, MD, ${ }^{3}$ and Ganesh Rao, MD ${ }^{1}$ \\ Departments of ${ }^{1}$ Neurosurgery, ${ }^{3}$ Diagnostic Radiology, ${ }^{4}$ Radiation Oncology, ${ }^{5}$ maging Physics, and ${ }^{6}$ Anesthesiology and \\ Perioperative Medicine, The University of Texas MD Anderson Cancer Center, Houston; and 'Department of Neurosurgery, \\ University of São Paulo, São Paulo, Brazil
}

\begin{abstract}
OBJECTIVE Image guidance for spinal procedures is based on 3D-fluoroscopy or CT, which provide poor visualization of soft tissues, including the spinal cord. To overcome this limitation, the authors developed a method to register intraoperative MRI (iMRI) of the spine into a neuronavigation system, allowing excellent visualization of the spinal cord. This novel technique improved the accuracy in the deployment of laser interstitial thermal therapy probes for the treatment of metastatic spinal cord compression.
\end{abstract}
METHODS Patients were positioned prone on the MRI table under general anesthesia. Fiducial markers were applied on the skin of the back, and a plastic cradle was used to support the MRI coil. T2-weighted MRI sequences of the re- gion of interest were exported to a standard navigation system. A reference array was sutured to the skin, and surface matching of the fiducial markers was performed. A navigated Jamshidi needle was advanced until contact was made with the dorsal elements; its position was confirmed with intraoperative fluoroscopy prior to advancement into a target in the epidural space. A screenshot of its final position was saved, and then the Jamshidi needle was exchanged for an MRI-compatible access cannula. MRI of the exact axial plane of each access cannula was obtained and compared with the corresponding screenshot saved during positioning. The discrepancy in millimeters between the trajectories was measured to evaluate accuracy of the image guidance

RESULTS Thirteen individuals underwent implantation of 47 laser probes. The median absolute value of the discrepancy between the location predicted by the navigation system and the actual position of the access cannulas was 0.7 $\mathrm{mm}$ (range 0-3.2 mm). No injury or adverse event occurred during the procedures.

CONCLUSIONS This study demonstrates the feasibility of image guidance based on MRI to perform laser interstitial thermotherapy of spinal metastasis. The authors' method permits excellent visualization of the spinal cord, improving safety and workflow during laser ablations in the epidural space. The results can be extrapolated to other indications, including biopsies or drainage of fluid collections near the spinal cord.

https://thejns.org/doi/abs/10.3171/2016.9.SPINE16475

KEY WORDS spinal laser interstitial thermotherapy; image guidance; magnetic resonance images; oncology

$\mathrm{T}$ HE incorporation of image guidance technology facilitated the development of new techniques in the care of patients suffering from spinal metastasis. Image guidance was initially used to perform biopsies ${ }^{1}$ and was quickly adapted for placement of implants ${ }^{4}$ and to aid complex resections. ${ }^{18}$ Its incorporation into radiation delivery methods resulted in a new field within radiation oncology, called stereotactic body radiotherapy (SBRT). ${ }^{6}$ Over the last decade, development of new platforms such as 3D fluoroscopy, cone beam CT, and intraoperative CT have provided surgeons several advantages, including improvements in tissue visualization, accuracy of instrument navigation, and decrease of exposure of ionizing radiation to operating room personnel and to patients. . $^{14,17,19}$

Intraoperative MRI (iMRI) has been commonly used in neurosurgery as a valuable tool for a wide range of cranial procedures, including biopsies, ${ }^{16}$ tumor resections ${ }^{13}$ implantation of deep brain stimulators, ${ }^{7}$ and, more recently, laser ablation of epileptogenic foci ${ }^{8}$ and tumors. ${ }^{5,15,24}$ However, the utilization of iMRI to perform spinal procedures

ABBREVIATIONS AP = anteroposterior; cEBRT = conventional external beam radiotherapy; ESCC = epidural spinal cord compression; iMRI = intraoperative MRI; KPS = Karnofsky Performance Status; SINS = Spinal Neoplastic Instability Score; sLITT = spinal laser interstitial thermotherapy; SSRS = spinal stereotactic radiosurgery. SUBMITTED April 25, 2016. ACCEPTED September 22, 2016.

INCLUDE WHEN CITING Published online February 10, 2017; DOI: 10.3171/2016.9.SPINE16475. 


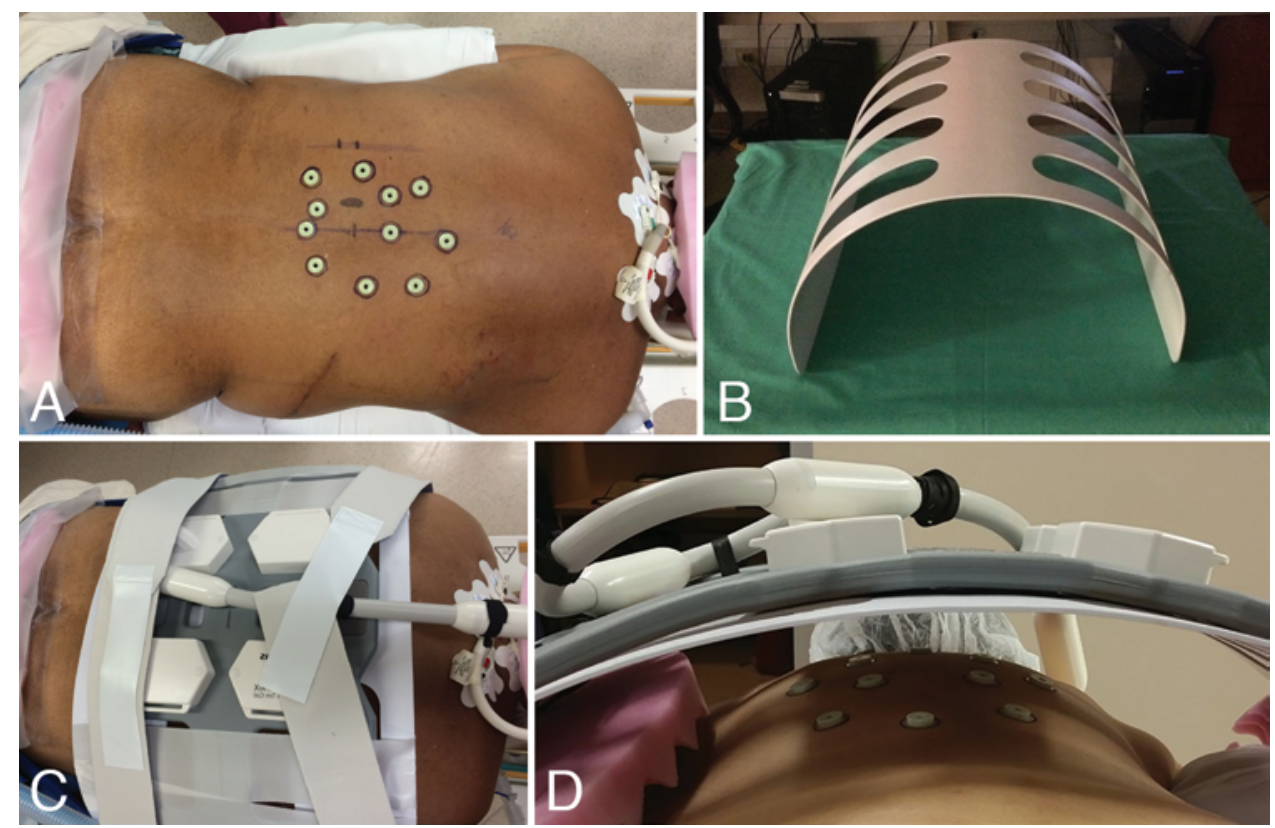

FIG. 1. Preparation for acquisition of intraoperative images for MRI-based image guidance. The patient is positioned prone on the MRI transfer table and standard fiducial markers placed overlying the region of interest (A). An MRI-compatible plastic cradle (B) is used. The MRI coil is taped over the cradle and placed over the fiducials $(\mathbf{C})$. Note that the cradle prevents contact with the coil and the fiducials (D), avoiding distortion of the position of the fiducials during manipulation of the patient into and out of the MRI scanner. Figure is available in color online only.

has been very limited, with just a few reports evaluating its feasibility in surgical decompressions ${ }^{23}$ and fenestration of intradural cysts. ${ }^{20,21}$

Here, we describe the use of image guidance for spinal laser interstitial thermotherapy (sLITT). ${ }^{12,22}$ This is the first report of utilization of iMRI to provide image guidance for placement of laser probes in the epidural space for treatment of spinal metastasis. Our results show that implementation of minor modifications in hardware allows this technique to be used with any iMRI, expanding the applicability of laser ablations and MRI-guided biopsies in soft tissue.

\section{Methods}

\section{Patient Population and Evaluation}

This single-institution retrospective analysis was conducted under an IRB-approved protocol. Patients who underwent placement of laser catheters with image guidance based on intraoperative MRI during sLITT between July 2015 to December 2015 were identified for this study, and their data were retrospectively reviewed. All individuals had documented spinal metastasis from histologies considered to have unfavorable response to conventional external beam radiotherapy (cEBRT). The epidural extension of the tumor was scored based on preoperative MRI on axial T2-weighted or postcontrast T1-weighted images according to the method described by Bilsky et al. ${ }^{3}$ Spinal stability was assessed by using the Spinal Instability Neoplastic Score (SINS) as described by Fisher et al. ${ }^{9}$ All cases were presented in a multidisciplinary tumor board conference with neurosurgery, radiation oncology, and radiology, during which consensus was reached that separa- tion of the tumor was needed prior to spinal stereotactic radiosurgery (SSRS). The utilization of the hardware and software in the patients whose data were reviewed in this report using MR images for spinal navigation is investigational and corresponds to an off-label use of such technology. Operative data included the iMRI and fluoroscopic images and the screen shots from the navigation system. All images used for analysis were deidentified. The radiologist collaborating on the study (B.A.) performed the measurements between the closest point of the dura in relation to the predicted and final trajectory of fiber implantation as described below.

\section{Image Guidance Technique for Placement of the Laser Probe in the Epidural Space}

The procedure was performed inside the iMRI (Brainlab Inc.). After general endotracheal anesthesia was induced, the patient was positioned prone over gel rolls placed in parallel along the body axis with the arms tucked to the side over the iMRI transfer table. A fluoroscope (Siemens) was positioned inside the room at a safe distance from the MRI unit to obtain anteroposterior and lateral fluoroscopic images. The level to be treated was localized, and standard fiducial markers (Izi Medical Products) were placed on the skin overlying the operative level (Fig. 1A). A Siemens body matrix coil was taped over a plastic cradle (Fig. 1B) and placed over the patient, with care taken to avoid touching the underlying skin and fiducials (Fig. 1C and D). The patient was transferred to the MRI scanner and a T2-weighted sequence of the region of interest was obtained. The images were transferred to the workstation of the image guidance system (Brainlab, Inc.), where a 3D 


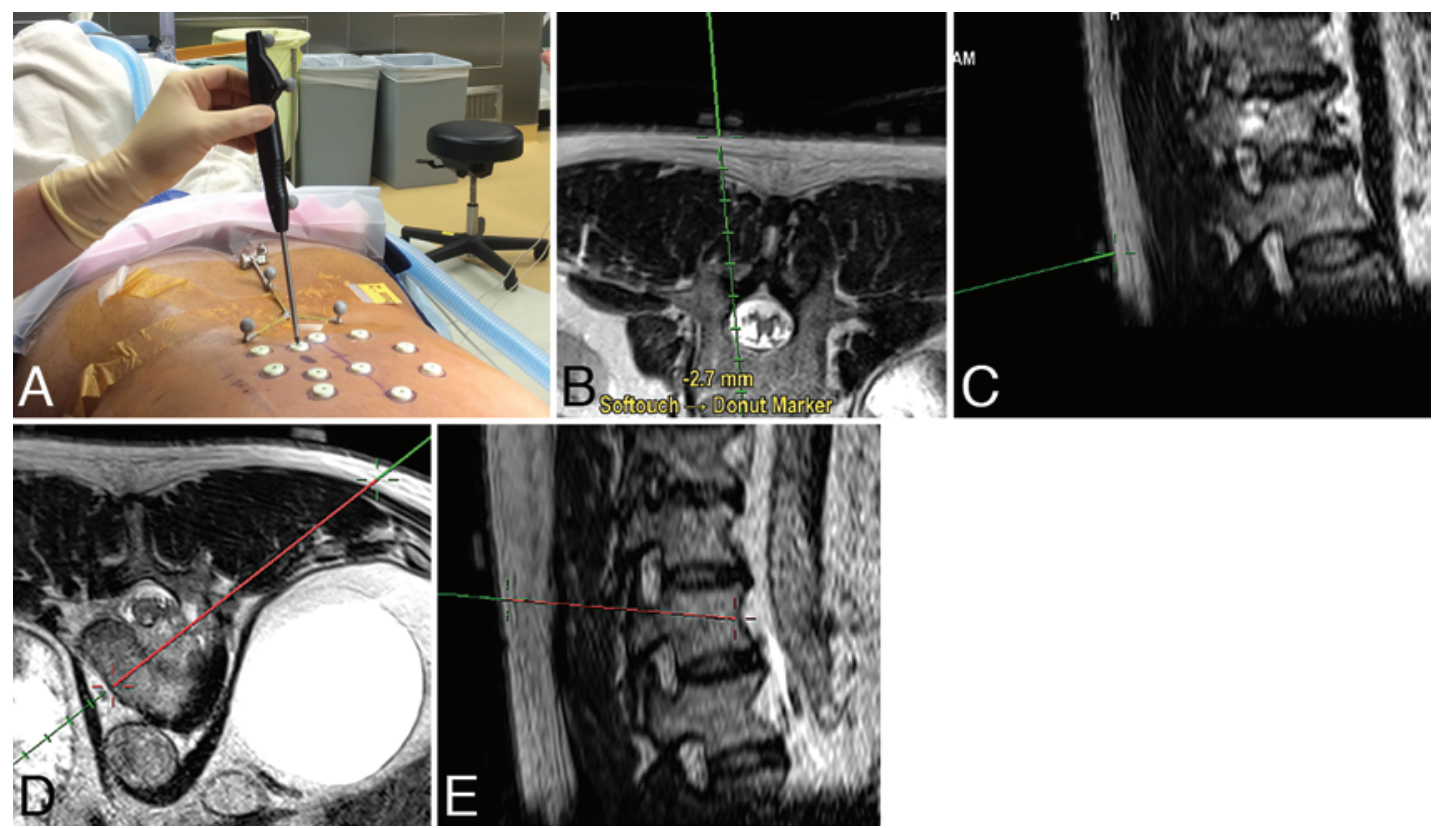

FIG. 2. Verification of surface accuracy of MRI-based image guidance. A: Intraoperative picture demonstrating the surgeon holding the navigation wand in the surface of the patient skin inside a fiducial marker. B and C: Axial (B) and sagittal (C) screenshots of the navigation monitor note the perfect correlation between the predicted position of the virtual wand with the actual position in the surface of the skin inside the fiducial. D and E: Axial (D) and sagittal $(E)$ screenshots of the inline views with a 7-cm offset used for trajectory planning. Figure is available in color online only.

model of the spinal segment containing the fiducials was created. A reference point was added in the center of each fiducial marker in contact with the patient's skin, and this plan was exported to the navigation system. The patient was removed from the MRI magnet, and the transfer table was repositioned in the safe zone outside of the high-power magnetic field in front of the $\mathrm{C}$-arm. The skin adjacent to the fiducials was prepared, and a reference array was secured with stiches and adhesive drape. A surface match of the fiducials was performed, taking care to avoid displacement of the skin. Accuracy of the navigation was easily verified by placing the navigation probe inside the fiducials (Fig. 2A-C) and at the midline overlying an easily palpable spinous process. Once this initial verification was considered accurate by the surgeon, an offset of $70 \mathrm{~mm}$ is added and image guidance in the inline axial and the sagittal projections were used to identify the proper trajectory to reach the epidural tumor at an approximate distance of 5 to $6 \mathrm{~mm}$ from the edge of the dura mater (Fig. 2D and E). A navigated Jamshidi needle (DePuy Synthes) registered to the navigation system was advanced through the soft tissues until it reached the lamina of the vertebral body affected by tumor. If the lateral projection of the navigation image was compatible with the tactile sensation of touching bone, the needle was docked in the lamina. At this stage, an anteroposterior (AP) fluoroscopic image was obtained (Fig. 3), and the surgeon compared the predicted location of the needle with the current AP projection using the pedicle of the adjacent level as a reference for the limits of the spinal canal. If the accuracy was satisfactory, the needle was advanced toward the predefined target and a snapshot of its final position was saved. At this stage, the central trocar was removed, and a K-wire was placed through the Jamshidi needle, which was subsequently removed. The K-wire was used as a guide for placement of a nonferromagnetic plastic access cannula. Based on our experience, a distance of 5-6 $\mathrm{mm}$ around each side of the laser fiber generates temperatures high enough to induce tumor cell death. If more coverage was needed, additional access cannulas were placed in tandem at a $10-$ to $12-\mathrm{mm}$ distance to cover the craniocaudal extension of the epidural tumor. Lastly, an MRI-compatible titanium needle was inserted into the access cannula and docked into the residual bone of the vertebral body, and a sterile plastic bag was used to cover the needles (Fig. 4).

To obtain good quality of images for the MRI thermography, we used 2 standard Siemens body matrix MRI coils placed on each side of the patient's back, overlapping in the center and held by straps. The patient was placed into the MRI magnet and a T2-weighted HASTE sequence was used to localize the artifact of the titanium needles, which allowed final confirmation of their trajectory. The ablation of the epidural tumor was performed as previously described. ${ }^{22}$

\section{Final Accuracy of the Needle Positioning}

Once the navigated Jamshidi needle reached its final position, a negative offset enough to reach the skin level was added to the predicted location of the needle, and a screenshot of the navigation monitor was saved. This represented the trajectory predicted by the navigation software from the entry point in the skin, to the target in the epidural space. The Jamshidi needle was replaced by the cannula, and the patient was prepared for the localization 
scans. ${ }^{22}$ T2-weighted MRI sequences reconstructed to the exact axial plane of each plastic access cannula were obtained. These images were fused to the corresponding final screenshot of each predicted trajectory using the navigation software (Brainlab, Inc.). The shortest distance between the predicted and the actual fiber trajectory in relation to the dural edge at the level of the spinal canal was measured in millimeters (Fig. 5A and B). Radial error (Fig. 5) was determined by measuring the tangential distance between the predicted and the real fiber position in the same axial plane. Laser catheters overlapping the desired trajectory were considered on target, with a radial error of zero, as described by Attaar et al. ${ }^{2}$

\section{Statistical Analysis}

Statistical analyses were performed with SPSS (IBM Corp.), using paired Student t-tests for the measurement between the predicted and the real distance from the closest point of the probe to the dura. The Wilcoxon signed rank tests assessed the median changes in thickness of epidural tumor and change in the epidural spinal cord classification (ESCC) classification. A p value $\leq 0.05$ was accepted as statistically significant.

\section{Results}

A total of 13 patients, 8 men and 5 women, with a median age of 60 years (range 40-81 years), underwent percutaneous placement of a total of 47 laser catheters in multiple spine levels for the treatment of epidural tumor (Table 1). All cases were performed under general anesthesia in the prone position. No morbidity was associated with the placement of the laser catheters or the thermal ablation, and the median hospital stay was 1 day (range 1-10 days). A total of 5 patients underwent SSRS after the laser ablation, 1 patient had cEBRT, and 7 patients had sLITT as salvage therapy not followed by radiation. The median length of imaging follow-up was 4.4 months (range 1.6-6.1 months).

Four patients had spinal instability, with a SINS score higher than 12, and were treated with placement of percutaneous pedicle screws. In 3 of those cases, pedicle screw placement was performed at the same time as ablation, using image guidance based on MRI (Cases 4, 5, and 13). One patient required long-segment stabilization (Case 7), which was performed in a separate operation 1 day after the sLITT.

The degree of spinal cord compression before and after treatment was assessed by using the ESCC score. The median preoperative score was 2 (range 1c-3), which was significantly higher than the median score of $1 \mathrm{~b}$ (range $0-3)$ at 4 months posttreatment $(p=0.0008$, Wilcoxon signed-rank test).

\section{Evaluation of Accuracy}

The fiducial registration demonstrated excellent surface accuracy with a margin of error so low that we were not able to measure the discrepancy from the predicted location of the tip of the needle in the navigation screen and the real position in the surface of the skin, both on axial and sagittal planes (Fig. 2).

The predicted position by the navigation system was
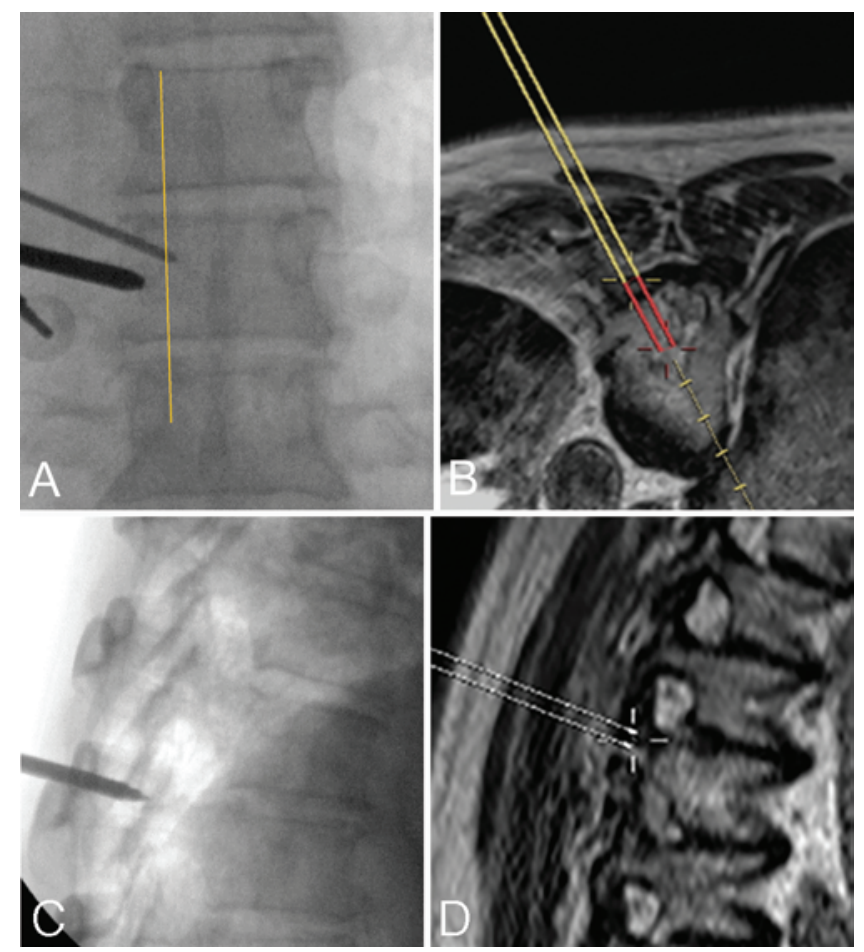

FIG. 3. Verification of accuracy at deep bone structures. A: Intraoperative fluoroscopy image obtained during advancement of the navigated Jamshidi needle. Note that the tip of the needle is lateral to the yellow line, which represents the medial border of the pedicles above and below the region of interest. In this example, a prior access needle has already been placed above the Jamshidi needle. B: Screenshot of the predicted position of the virtual needle in yellow, corresponding to the position depicted in Panel A. In this example, we placed a 3-cm offset (red) which allows predicting the final trajectory of the navigation. C: Lateral fluoroscopic image demonstrating the navigated needle positioned in the upper portion of the pedicle. D: Screenshot of the predicted position of the virtual needle demonstrating good correlation to the fluoroscopic image in Panel C. Figure is available in color online only.

not saved on 3 occasions; therefore, out of 47 successfully implanted catheters, we have analyzed the accuracy of 44 laser fibers. MR images provided excellent visualization of the spinal cord and the epidural tumor in all cases. The median distance from the dura predicted by the navigation was $6.6 \mathrm{~mm}$ (range $3.5-11.6 \mathrm{~mm}$ ), which was not different from the real median distance of the fiber of $6.7 \mathrm{~mm}$ (range 3.5-10.8 mm. p = 0.13, paired t-test; Table 2).

The median absolute value of the discrepancy in trajectory or radial error was $0.7 \mathrm{~mm}$ (range $0-3.2 \mathrm{~mm}$ ), which did not correlate with adverse events or failure in the local control of the treated tumor at 4.4 months' follow up.

\section{Discussion}

A multitude of spinal interventions are traditionally guided by conventional 2D fluoroscopic imaging, which has the advantages of portability, low cost, and compatibility with the sterile operative field. However, the image quality is subject to deterioration in patients with severe osteoporosis or morbid obesity, and, most importantly, complex oblique trajectories are not properly imaged in the $2 \mathrm{D}$ plane. Given these limitations, image guidance for 

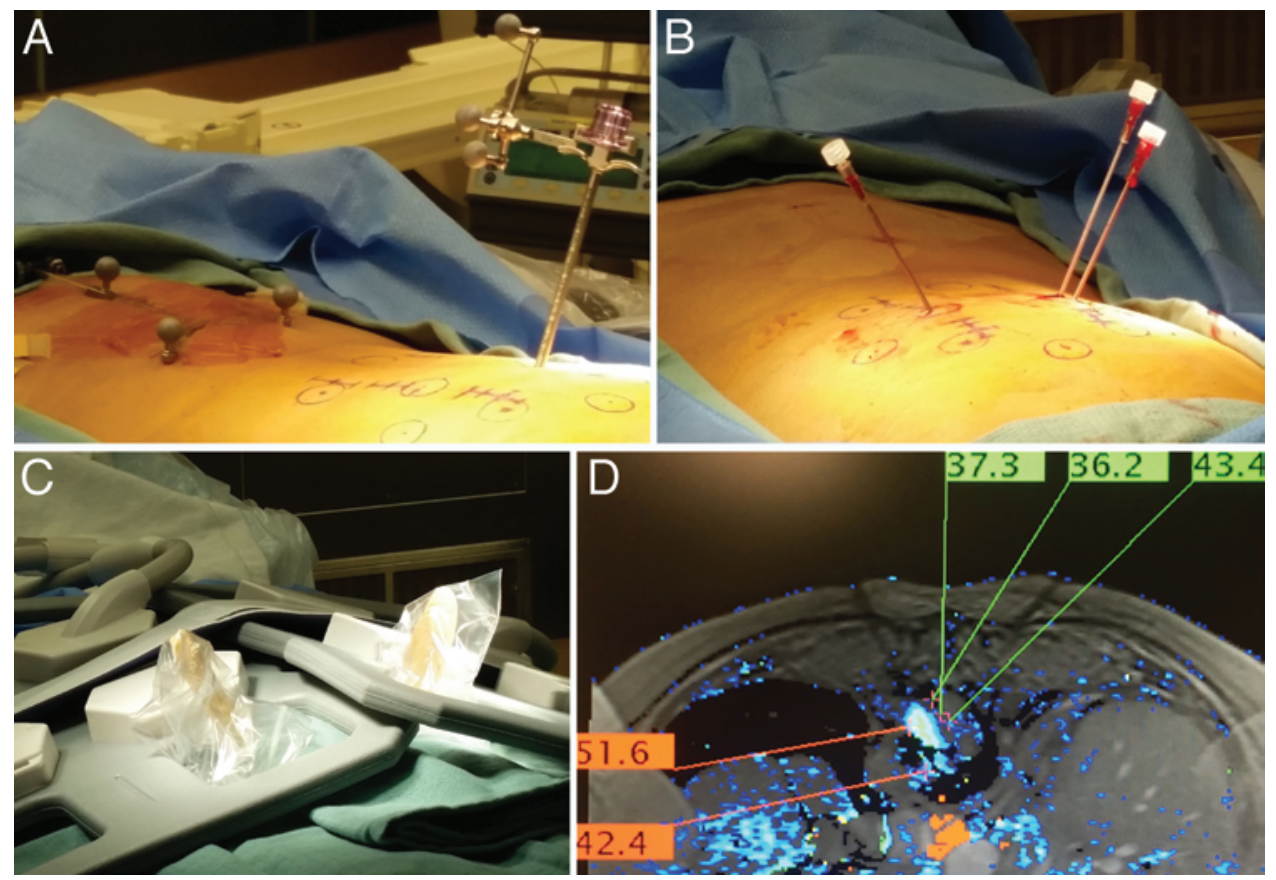

FIG. 4. Performance of the laser ablation. The navigated Jamshidi needle is advanced into the final position (A), and a screenshot is saved. The Jamshidi needle is exchanged for a plastic access cannula (B), and an MRI-compatible titanium needle is used to maintain its position until the placement of the laser fiber. The needles are covered with sterile technique and $2 \mathrm{MRI}$ coils are applied over the dorsal region (C). The patient is transferred to the MRI magnet for the laser ablation. An intraoperative image from the MRI thermography (D) showing the green boxes that indicate temperature on the epidural space and the red boxes that indicate the temperature near the fiber.

spinal procedures was developed based on CT images, which are ideal for delineation of bony structures, and this technique has become widely used for placement of posterior segmental instrumentation for which a series of surgical tools have been developed and adapted.
We have previously reported our method of placing laser catheters to perform thermal ablation of epidural tumors. ${ }^{22}$ Briefly, a preoperative CT (obtained with the patient supine) is uploaded to the navigation system and overlaid with an AP and lateral fluoroscopic image (obtained with
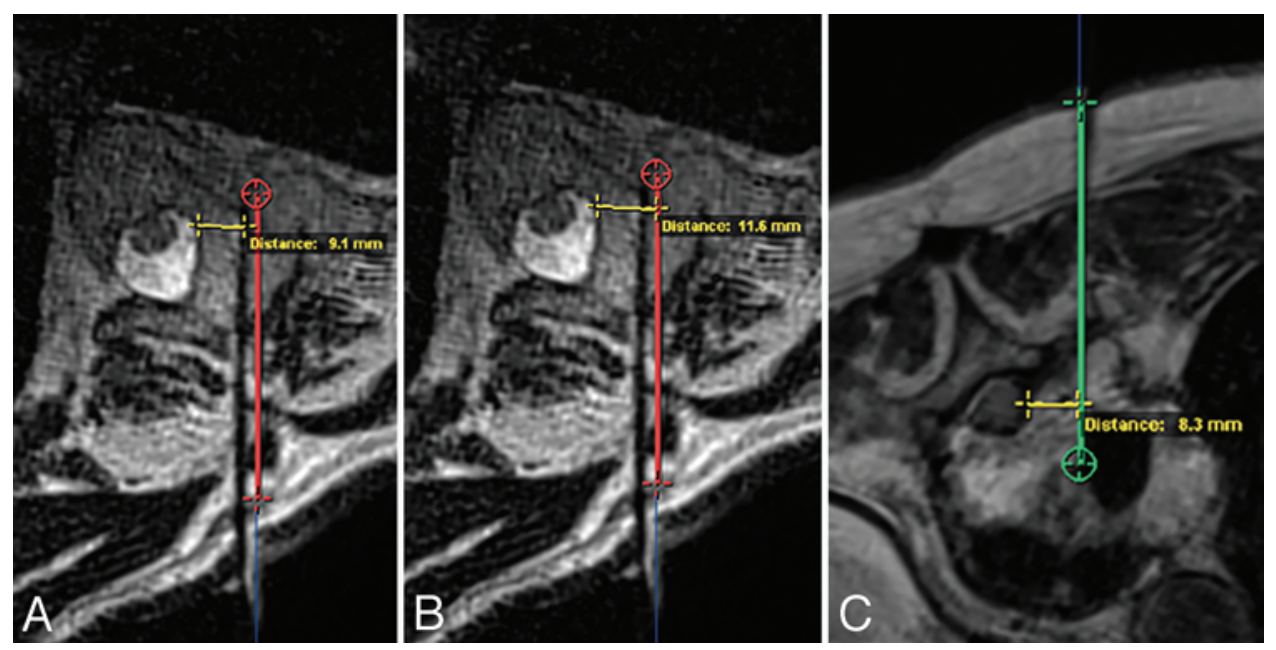

FIG. 5. Measurement of accuracy of fiber placement. The final screenshot of the position of the navigated Jamshidi needle is fused with the intraoperative MRI in the exact axial plane of the laser probe. A and B: Examples of fused images showing measurement of the closest distance between the actual position of the laser fiber $(A)$ and the closest distance between the dura and the predicted position of the navigated needle (B), which is measured in the same plane as the actual position. The radial error is the discrepancy between these measurements. In this example (Case 12), the 2 measured distances are, respectively, $9.1 \mathrm{~mm}$ and $11.6 \mathrm{~mm}$, and the radial error is $2.5 \mathrm{~mm}$. The red is an offset to demonstrate what would be the final position of the needle. The yellow portion is the real needle, which needs to be compared to the fluoroscopic image. C: Example of a perfect overlap between the predicted (green line) and the actual position of the laser fiber (Case 13), with a radial error of $0 \mathrm{~mm}$. 
TABLE 1. Demographic and clinical characteristics of patients enrolled in our study

\begin{tabular}{|c|c|c|c|c|c|c|c|c|c|c|}
\hline \multirow[b]{2}{*}{$\begin{array}{c}\text { Case } \\
\text { No. }\end{array}$} & \multirow{2}{*}{$\begin{array}{c}\text { Age } \\
\text { (yrs), } \\
\text { Sex }\end{array}$} & \multirow[b]{2}{*}{$\begin{array}{c}\text { Primary } \\
\text { Tumor }\end{array}$} & \multirow[b]{2}{*}{ Level } & \multirow{2}{*}{$\begin{array}{c}\text { Preop } \\
\text { KPS } \\
(\%)\end{array}$} & \multirow[b]{2}{*}{ SINS } & \multirow[b]{2}{*}{$\begin{array}{l}\text { LOS } \\
\text { (days) }\end{array}$} & \multicolumn{3}{|c|}{ Radiation Treatment } & \multirow[b]{2}{*}{ Remark } \\
\hline & & & & & & & Type & $\begin{array}{l}\text { Time From } \\
\text { Op (days) }\end{array}$ & $\begin{array}{l}\text { Dose } \\
\text { (Gy/Fr) }\end{array}$ & \\
\hline 1 & $76, M$ & Thyroid & T6-7 & 90 & 6 & 1 & NA & NA & NA & Prior cEBRT, SSRS \\
\hline 2 & $40, M$ & Colon & $\mathrm{T}-2$ & 70 & 8 & 2 & NA & NA & NA & Prior cEBRT, SSRS \\
\hline 3 & $63, M$ & RCC & T8-9 & 50 & 10 & 1 & NA & NA & NA & Prior cEBRT, SSRS \\
\hline 4 & $56, M$ & Lung & $\mathrm{T}-2$ & 60 & 14 & 5 & cEBRT & 4 & $30 / 10$ & Perc stab T1-3 \\
\hline 5 & $81, \mathrm{~F}$ & Lung & L-1 & 40 & 13 & 5 & SSRS & 10 & $27 / 3$ & Prior cEBRT, perc stab T12-L2 \\
\hline 6 & $46, \mathrm{~F}$ & Thyroid & T-2 & 80 & 7 & 1 & SSRS & 3 & $24 / 1$ & \\
\hline 7 & $63, \mathrm{~F}$ & Lung & T4-6 & 40 & 16 & 10 & NA & NA & NA & Prior cEBRT, perc stab T2-3 to T7-8 \\
\hline 8 & $68, \mathrm{~F}$ & Melanoma & $\mathrm{T}-4$ & 80 & 6 & 1 & NA & NA & NA & Prior SSRS \\
\hline 9 & $58, M$ & RCC & T2-3 & 90 & 6 & 1 & SSRS & 6 & $27 / 3$ & \\
\hline 10 & $53, M$ & Lung & $\mathrm{T}-2$ & 70 & 10 & 1 & NA & NA & NA & Prior cEBRT, SSRS \\
\hline 11 & $64, \mathrm{M}$ & Thyroid & $\mathrm{T}-2$ & 80 & 8 & 1 & SSRS & 6 & $18 / 1$ & \\
\hline 12 & $52, \mathrm{M}$ & SCC tongue & T7-8 & 80 & 6 & 1 & SSRS & 4 & $27 / 3$ & \\
\hline 13 & $60, \mathrm{~F}$ & Thyroid & $\mathrm{T}-11$ & 40 & 13 & 4 & NA & NA & NA & Perc stab T10-L2, prior cEBRT \\
\hline
\end{tabular}

cEBRT = conventional external beam radiotherapy; $L O S=$ length of stay in hospital; NA = not available; perc stab = percutaneous stabilization; RCC = renal cell carcinoma; SCC = squamous cell carcinoma; SINS = Spinal Instability Neoplastic Score; SSRS = spinal stereotactic radiosurgery.

the patient in the operative position). Computer software performs the alignment and fusion of images, and 2D and $3 \mathrm{D}$ models of the region of interest are created. This method has 2 major shortcomings: 1) Surface inaccuracy: As the preoperative CT images are acquired in prone position, the weight of the body compresses the soft tissue of the dorsal region against the spine. When patient is positioned for surgery, such compression is relieved, resulting in a significant mismatch, precluding verification of accuracy of the image guidance. 2) Poor visualization of the spinal cord, especially in cases of significant epidural compression.

The utilization of MRI as guidance for surgical procedures in the spine is very limited, and few reports have been published on it. ${ }^{20,21,23} \mathrm{~A}$ significant limitation of this method is the incompatibility of surgical instruments since surgery occurs inside or near a high-power magnetic field. Fritz et al. ${ }^{11}$ described the use of augmented reality in which intraoperative MR images are uploaded to planning software. A frame is positioned over the region of interest and a liquid crystal display is used for passive projection of the MR images in a semitransparent mirror over the dorsal region of the subject. The surgeon can guide a bone needle based on the entry point and the target projected on the patient skin. The final error of this complex setup was $6.1 \pm 1.9 \mathrm{~mm}$ on the target for vertebroplasties ${ }^{10}$ and $4.3 \pm$ $1.2 \mathrm{~mm}$ for the vertebral body biopsies..$^{11}$ These were both experimental studies with cadavers, and the cost and the feasibility of this method is as yet unknown.

Using MRI for placement of the laser catheters provides several advantages over our previously described technique: 1) verification of accuracy of image guidance on the skin surface; 2) planning of the proper trajectory from the surface, minimizing the length of the skin incisions; and 3) excellent visualization of the spinal cord, even in cases of severe compression, enabling safe positioning of the catheters in the epidural space.
Our method takes advantage of standard fiducial markers, which are placed over the dorsal region. As a requirement for registration, these markers have to be visible in the scan; therefore, the MRI coil has to be positioned above those fiducials. As the skin of the dorsal region is very loose, it is imperative to avoid displacement of the markers after the MRI is completed, since even minor changes in position will result in inaccurate registration. To overcome these limitations, we introduced the use of a plastic cradle to support the MRI coil above the fiducials, avoiding contact with them. This step is extremely important, and we recommend careful observation of the cradle as the patient is transported into and out of the MRI scanner.

It has been our experience that the ideal MRI sequence for fiducial registration is T2-weighted without fat saturation. This sequence allows excellent visualization of the CSF and the spinal cord and is less susceptible to motion degradation due to respiratory movements. Once the registration sequence is exported to the planning software, we recommend that the reference point for registration be placed $1 \mathrm{~mm}$ below the fiducial, to compensate for the displacement of the dorsal skin associated with the "wiggling" of the registration wand at the time of surface matching of the fiducials.

The iMRI room is designed to allow the use of common instruments and equipment. Our institution has a very strict policy for safety, requiring all ferromagnetic non-MRI compatible instruments to be counted and positioned outside the high-power magnetic field, which is outlined in the floor. For the implantation of the laser catheters, the patient needs to be removed from the MRI magnet, since all supporting equipment (fluoroscope, anesthesia cart, navigation hardware, and surgical instruments) are not MRI compatible. We take advantage of the iMRI transfer table, which allows minimal manipulation of the patient during this process. Registration of the fiducials requires placement 
TABLE 2. Details of treatment parameters in 13 patients in whom sLITT was performed

\begin{tabular}{|c|c|c|c|c|c|c|c|}
\hline \multirow{2}{*}{$\begin{array}{l}\text { Case } \\
\text { No. }\end{array}$} & \multirow{2}{*}{$\begin{array}{l}\text { Preop } \\
\text { ESCC }\end{array}$} & \multirow{2}{*}{$\begin{array}{l}\text { Postop } \\
\text { ESCC }\end{array}$} & \multirow{2}{*}{$\begin{array}{l}\text { No. of } \\
\text { Fibers }\end{array}$} & \multicolumn{2}{|c|}{ Mean Distance From Fibers to Dura (mm) } & \multirow{2}{*}{$\begin{array}{l}\text { Average Radial } \\
\text { Error (mm) }\end{array}$} & \multirow{2}{*}{$\begin{array}{l}\text { Time to Imaging } \\
\text { Follow-Up (mos) }\end{array}$} \\
\hline & & & & Predicted & Actual & & \\
\hline 1 & 3 & $1 \mathrm{~b}$ & 4 & 7.0 & 7.7 & 0.7 & 5.4 \\
\hline 2 & 2 & 1c & 3 & 4.1 & 5.1 & 1.0 & 5.4 \\
\hline 3 & 3 & 3 & 8 & 7.5 & 7.1 & 0.7 & 4.3 \\
\hline 4 & 3 & $1 b$ & 1 & 6.2 & 7.4 & 1.2 & 2.0 \\
\hline 5 & 2 & 2 & 3 & 7.0 & 6.6 & 0.4 & 2.1 \\
\hline 6 & 2 & 0 & 3 & 7.1 & 8.4 & 1.4 & 6.1 \\
\hline 7 & 2 & NA & 3 & 5.8 & 5.8 & 0.0 & NA \\
\hline 8 & 2 & $1 b$ & $4^{*}$ & 10.4 & 9.9 & 0.5 & 5.2 \\
\hline 9 & $1 \mathrm{c}$ & 0 & $5^{*}$ & 7.5 & 6.4 & 1.2 & 4.6 \\
\hline 10 & 2 & 3 & $3^{*}$ & 6.2 & 7.0 & 0.8 & 2.1 \\
\hline 11 & 2 & $1 b$ & 2 & 7.1 & 6.7 & 0.5 & 4.6 \\
\hline 12 & 2 & $1 b$ & 5 & 8.0 & 5.3 & 2.7 & 3.4 \\
\hline 13 & 3 & $1 a$ & 3 & 6.5 & 6.4 & 0.1 & 1.6 \\
\hline Median & 2 & $1 b$ & 3 & 7.0 & 6.7 & 0.7 & 4.4 \\
\hline
\end{tabular}

* One fiber excluded from analysis due to lack of corresponding predicted position.

of a reference array, which we suture to the dorsal skin at least 12 inches away from the fiducial markers. We avoid performing a skin incision to attach the array to a spinous process, since this maneuver stretches the skin and creates displacement of the fiducials. Similarly, surgical draping needs to be loose and not attached to the skin, to avoid displacement of the fiducials or the array. Lastly, during fiber placement, it is imperative that the surgeon and the assistant not touch the dorsal region of the patient to avoid displacement of the array and complete loss of accuracy.

Once the navigated Jamshidi needle is registered, we double-check its accuracy before it is advanced toward the spinal cord. It has been our experience that surface accuracy predicts accuracy of the needle at the target. However, we do obtain an AP fluoroscopic image to confirm needle position based on the pedicle of an uninvolved adjacent level as a reference for the limits of the spinal canal.

We have performed placement of 47 laser fibers without any morbidity. MRI guidance has significantly improved our workflow since visualization of the spinal cord has added an extra degree of confidence, reducing the need for additional confirmatory fluoroscopic images during needle advancement. We estimate an intraoperative timing of implantation of 15 minutes per fiber. Our results estimate a median absolute radial error or discrepancy between the predicted and the actual trajectory of the navigated needle to be $0.7 \mathrm{~mm}$ (range $0-3.2 \mathrm{~mm}$ ), which is similar to the data reported for stereotactic placement in cranial procedures. ${ }^{2}$ Since we aim for a distance of 5-6 mm lateral to the dura, this margin of error is acceptable. We use the inline or trajectory axial view to track the needle as it is advanced toward the target. Once the needle is docked on the lamina, we release the navigated needle to make adjustments to the trajectory if necessary. We recommend against forcing corrections as the needle is advanced. Since each fiber is positioned freehand, we believe that most of our discrepancies between the predicted and the real position of the needle occurred because we had the impression of a dif- ferent trajectory as we were manipulating the needle to the desired angle during advancement through the lamina or pedicle. Once we reached the final position, release of the needle correlated with a slightly different angle, therefore explaining some of the cases of higher radial error. Another source of error is the unnoticed development of a subcutaneous hematoma or tension of the skin as the needle is introduced in oblique angles, displacing the reference array. This problem would be easily overcome with development of a nonferromagnetic array, which could be securely attached to the spinous process and scanned in conjunction with the fiducials prior to registration.

Our method allowed reregistration of the fiducials, since we outlined them with a marking pen prior to the registration scan. This added the ability to use navigation for placement of the posterior segmental instrumentation after the laser ablation was completed. Although this task could be easily achieved with fluoroscopy, navigation helped expedite the workflow inside the iMRI room.

An evaluation of the effectiveness of the laser ablations in controlling the epidural tumor is beyond the scope of this report and will be the focus of future publications. In the short follow-up of our series, we have demonstrated good clinical local control in 11 of 13 patients, in whom the median Bilsky ESCC score decreased from 2 to $1 \mathrm{~b}$. A total of 7 patients had SLITT as a salvage procedure after failure of prior surgical and radiation treatments. In 2 of those 7 cases, the patients had no improvement in ESCC but remained neurologically stable during the study interval. All cases were associated with a short hospital stay, and we believe patients were able to resume oncological treatment faster than if they had undergone standard open surgical decompression and stabilization procedures, especially the individuals with a Karnofsky Performance Status (KPS) lower than $60 \%$ in whom the added surgical morbidity would be excessive. 


\section{Conclusions}

We demonstrate the feasibility and accuracy of using intraoperative MR images for image guidance in spinal procedures. To our knowledge, this is the first report of such use, which, in our hands, has been proven to be accurate and reliable for placement of laser catheters and facilitated the placement of percutaneous stabilization in the same procedure. We believe this method can be used for other applications, including biopsies and drainage of cysts or abscesses.

\section{References}

1. Adapon BD, Legada BD Jr, Lim EV, Silao JV Jr, DalmacioCruz A: CT-guided closed biopsy of the spine. J Comput Assist Tomogr 5:73-78, 1981

2. Attaar SJ, Patel NV, Hargreaves E, Keller IA, Danish SF: Accuracy of laser placement with frameless stereotaxy in magnetic resonance-guided laser-induced thermal therapy. Neurosurgery 11:554-563, 2015

3. Bilsky MH, Laufer I, Fourney DR, Groff M, Schmidt MH, Varga PP, et al: Reliability analysis of the epidural spinal cord compression scale. J Neurosurg Spine 13:324-328, 2010

4. Bourgeois AC, Faulkner AR, Bradley YC, Pasciak AS, Barlow PB, Gash JR, et al: Improved accuracy of minimally invasive transpedicular screw placement in the lumbar spine with 3-dimensional stereotactic image guidance: a comparative meta-analysis. J Spinal Disord Tech 28:324-329, 2015

5. Carpentier A, McNichols RJ, Stafford RJ, Itzcovitz J, Guichard JP, Reizine D, et al: Real-time magnetic resonance-guided laser thermal therapy for focal metastatic brain tumors. Neurosurgery 63 (1 Suppl 1):ONS21-ONS29, 2008

6. Chang BK, Timmerman RD: Stereotactic body radiation therapy: a comprehensive review. Am J Clin Oncol 30:637644, 2007

7. Cui Z, Pan L, Song H, Xu X, Xu B, Yu X, et al: Intraoperative MRI for optimizing electrode placement for deep brain stimulation of the subthalamic nucleus in Parkinson disease. J Neurosurg 124:62-69, 2016

8. Curry DJ, Gowda A, McNichols RJ, Wilfong AA: MR-guided stereotactic laser ablation of epileptogenic foci in children. Epilepsy Behav 24:408-414, 2012

9. Fisher CG, DiPaola CP, Ryken TC, Bilsky MH, Shaffrey CI, Berven SH, et al: A novel classification system for spinal instability in neoplastic disease: an evidence-based approach and expert consensus from the Spine Oncology Study Group. Spine (Phila Pa 1976) 35:E1221-E1229, 2010

10. Fritz J, U-Thainual P, Ungi T, Flammang AJ, Kathuria S, Fichtinger G, et al: MR-guided vertebroplasty with augmented reality image overlay navigation. Cardiovasc Intervent Radiol 37:1589-1596, 2014

11. Fritz J, U-Thainual P, Ungi T, Flammang AJ, McCarthy EF, Fichtinger G, et al: Augmented reality visualization using image overlay technology for MR-guided interventions: cadaveric bone biopsy at 1.5 T. Invest Radiol 48:464-470, 2013

12. Ghia AJ, Rebueno NC, Li J, Brown PD, Rhines LD, Tatsui $\mathrm{CE}$ : The use of image guided laser interstitial thermotherapy to supplement spine stereotactic radiosurgery to manage metastatic epidural spinal cord compression: proof of concept and dosimetric analysis. Pract Radiat Oncol 6:e35-e38, 2016

13. Hatiboglu MA, Weinberg JS, Suki D, Rao G, Prabhu SS, Shah K, et al: Impact of intraoperative high-field magnetic resonance imaging guidance on glioma surgery: a prospective volumetric analysis. Neurosurgery 64:1073-1081, 2009

14. Kim TT, Drazin D, Shweikeh F, Pashman R, Johnson JP: Clinical and radiographic outcomes of minimally invasive percutaneous pedicle screw placement with intraoperative
CT (O-arm) image guidance navigation. Neurosurg Focus 36(3):E1, 2014

15. Missios S, Bekelis K, Barnett GH: Renaissance of laser interstitial thermal ablation. Neurosurg Focus 38(3):E13, 2015

16. Moriarty TM, Quinones-Hinojosa A, Larson PS, Alexander E III, Gleason PL, Schwartz RB, et al: Frameless stereotactic neurosurgery using intraoperative magnetic resonance imaging: stereotactic brain biopsy. Neurosurgery 47:1138-1146, 2000

17. Rahmathulla G, Nottmeier EW, Pirris SM, Deen HG, Pichelmann MA: Intraoperative image-guided spinal navigation: technical pitfalls and their avoidance. Neurosurg Focus 36(3):E3, 2014

18. Smitherman SM, Tatsui CE, Rao G, Walsh G, Rhines LD: Image-guided multilevel vertebral osteotomies for en bloc resection of giant cell tumor of the thoracic spine: case report and description of operative technique. Eur Spine $\mathbf{J}$ 19:1021-1028, 2010

19. Tabaraee E, Gibson AG, Karahalios DG, Potts EA, Mobasser JP, Burch S: Intraoperative cone beam-computed tomography with navigation $(\mathrm{O}-\mathrm{ARM})$ versus conventional fluoroscopy (C-ARM): a cadaveric study comparing accuracy, efficiency, and safety for spinal instrumentation. Spine (Phila Pa 1976) 38:1953-1958, 2013

20. Takahashi S, Morikawa S, Saruhashi Y, Matsusue Y, Kawakami M: Percutaneous transthoracic fenestration of an intramedullary neurenteric cyst in the thoracic spine with intraoperative magnetic resonance image navigation and thoracoscopy. J Neurosurg Spine 9:488-492, 2008

21. Takahashi S, Saruhashi Y, Odate S, Matsusue Y, Morikawa S: Percutaneous aspiration of spinal terminal ventricle cysts using real-time magnetic resonance imaging and navigation. Spine (Phila Pa 1976) 34:629-634, 2009

22. Tatsui CE, Stafford RJ, Li J, Sellin JN, Amini B, Rao G, et al: Utilization of laser interstitial thermotherapy guided by real-time thermal MRI as an alternative to separation surgery in the management of spinal metastasis. J Neurosurg Spine 23:400-411, 2015

23. Woodard EJ, Leon SP, Moriarty TM, Quinones A, Zamani AA, Jolesz FA: Initial experience with intraoperative magnetic resonance imaging in spine surgery. Spine (Phila Pa 1976) 26:410-417, 2001

24. Yeniaras E, Fuentes DT, Fahrenholtz SJ, Weinberg JS, Maier F, Hazle JD, et al: Design and initial evaluation of a treatment planning software system for MRI-guided laser ablation in the brain. Int J CARS 9:659-667, 2014

\section{Disclosures}

Dr. Li reports receipt of support for non-study-related clinical or research effort from Medtronic. Dr. Rhines reports a consultant relationship with Stryker and Globus.

\section{Author Contributions}

Conception and design: Tatsui, Suki. Acquisition of data: Tatsui. Analysis and interpretation of data: Tatsui, Nascimento, Amini, Kumar, Rao. Drafting the article: Tatsui, Nascimento. Critically revising the article: Nascimento, Suki, Amini, Li, Ghia, Thomas, Stafford, Rhines, Cata, Kumar, Rao. Reviewed submitted version of manuscript: Tatsui, Rhines, Rao. Approved the final version of the manuscript on behalf of all authors: Tatsui. Statistical analysis: Suki. Administrative/technical/material support: Stafford.

\section{Correspondence}

Claudio E. Tatsui, Department of Neurosurgery, The University of Texas MD Anderson Cancer Center, 1515 Holcombe Blvd., Unit 442, Houston, TX 77030. email: cetatsui@mdanderson.org. 